\title{
Spatial Transformations in the Withdrawal Response of the Tail in Intact and Spinalized Rats
}

\author{
Corey L. Cleland and Ross E. Bauer \\ Department of Biology, James Madison University, Harrisonburg, Virginia 22807
}

Previous studies of spatial transformations between sensory input and motor output in escape responses have suggested two alternative patterns of spatial integration. The continuous pattern corresponds to withdrawal movements directed $180^{\circ}$ away from the location of the stimulus, whereas the categorical pattern corresponds to movements that are biased toward a limited number of preferred directions. The goal of these experiments was to determine which pattern best describes the tail withdrawal response in spinalized and intact rats by applying pinpoint heat stimuli at eight points distributed circumferentially around the tail and measuring the direction and speed of the resulting withdrawal response. Our results are consistent with a novel, hybrid continuous-categorical movement strategy. In the spinalized animal, responses were primarily away from the stimulus (the continuous component) but exhibited a pronounced ventral bias (the categorical component). In addition, stimuli delivered to the ventral surface, which would be expected to evoke a dorsally directed response (opposite to the preferred ventral direction), instead evoked responses that were markedly variable and clustered in the left and right directions. Intact rats showed a similar pattern of response, except reversed; the bias was in the dorsal direction, and the response to dorsal stimulation was now highly variable and lateral in direction. These results reveal a complex tail withdrawal strategy that is modulated by descending supraspinal pathways to adapt the response to the biomechanical and environmental constraints imposed on movement of the rat tail.

Key words: spinal; reflex; pain; nociception; thermal; flexion
Movement requires that the CNS transform sensory information into spatially and temporally coordinated activation of muscles (Bizzi et al., 1991). In mammals, the large number of degrees of freedom represented by the hundreds of muscles and joints represent a significant computational burden for the production of accurate, short-latency responses to sensory information (Bernstein, 1967; Saltzman, 1979). Because simple reflexes can display significant spatial complexity but are more experimentally tractable, they offer an ideal model for investigating spatial transformations. In particular, the withdrawal reflex, in which a tissuedamaging or potentially tissue-damaging sensory stimulus evokes a withdrawal response, is a spinal reflex that exhibits complex spatial integration, thus providing an appropriate model to elucidate general principles of spatial sensorimotor transformations (Sherrington, 1910; Krasne and Wine, 1984; Eaton and Emberley, 1991; Norekian and Satterlie, 1996; Lewis and Kristan, 1999).

Previous studies of escape and withdrawal responses suggested two alternative patterns of spatial integration (Lewis and Kristan, 1999). The continuous pattern corresponds to withdrawal movements that are directed $180^{\circ}$ away from the location of the stimulus. In contrast, the categorical pattern corresponds to movement that is biased toward a limited number of preferred directions. For example, the early suggestion of Sherrington (1910) that stimuli applied anywhere to the leg evoke identical flexion responses would be an example of a categorical pattern with one category. However, his recognition that the pattern of

\footnotetext{
Received Oct. 9, 2001; revised Jan. 30, 2002; accepted March 11, 2002.

This work was funded by National Institutes of Health Grant NS32261 and James Madison University. We thank Andrew Hawkins, Brady Allen, Katherine Johnson, and Steve Gentile for technical assistance.

Correspondence should be addressed to Corey Cleland, Department of Biology, James Madison University, Harrisonburg, VA 22807. E-mail: clelancl@jmu.edu. Copyright (C) 2002 Society for Neuroscience $0270-6474 / 02 / 225265-06 \$ 15.00 / 0$
}

response varied somewhat with stimulus location, known as "local sign", is consistent with a continuous pattern. In mammals there is conflicting evidence for the two alternative patterns. Both Sherrington (1910) and Carstens and Wilson (1993) had results that were on balance more consistent with a categorical pattern. In contrast, Hagbarth (1952) showed clear examples of both excitation and inhibition in the same muscle, depending on the location of stimulus, which is consistent with a continuous pattern. Similarly, Schouenborg and Kalliomaiki (1990) and Schouenborg et al. $(1992,1994)$ showed that muscles excited by stimulation of the skin would be expected to withdraw the skin away from the stimulus, again more consistent with a continuous pattern.

The goal of these experiments was to determine whether the continuous or categorical pattern best describes the tail withdrawal response in spinalized and intact rats. The tail was chosen because the radial symmetry of the surface is well matched to studying spatial transformations and there is extensive literature in the pain field on heat-evoked "tail flick" responses (Grossman et al., 1982; Dubner, 1994). Our results demonstrate that the withdrawal reflex of the rat tail uses a unique strategy that is a hybrid of continuous and categorical patterns of movement and that is qualitatively altered by supraspinal control. These results suggest a novel form of spatial sensorimotor integration that may be applicable to other models of spatial transformations.

\section{MATERIALS AND METHODS}

Rats (male, Sprague Dawley, 400-450 gm; Harlan, Indianapolis, IN) were placed in a conventional cylindrical, acrylic restraining tube and then positioned vertically (Newport, Irvine, CA) above the testing apparatus (see Fig. 1). The rat was allowed 45 min to acclimatize before the experiment.

Pinpoint heat stimuli were delivered with a projection lamp (Fuji, CXL/CXR, $8 \mathrm{~V} 50 \mathrm{~W}$ ) focused with two lenses into a $1 \mathrm{~mm}$ spot. Low-intensity illumination (2.8 V AC) was used to aim the light, and high 
intensity ( $8.2 \mathrm{~V} \mathrm{AC}$ ) was used to deliver heat to eight spots evenly distributed circumferentially around the tail in $45^{\circ}$ increments (corresponding to $3 \mathrm{~mm}$ spacing) and aligned with the ventral, dorsal, left, and right sides of the rat, and four intermediate positions. The rat was rotated, and the heat source remained fixed. Each location was marked with a $1 \mathrm{~mm}$ black ink spot to insure reproducibility and enhance local heat absorption. Withdrawal of the tail terminated the stimulus, minimizing discomfort to the rat.

The spread of heating of the tail was measured with a miniature, surface thermocouple (MT-4, $0.125 \mathrm{sec}$ time constant; Physitemp, Clifton, $\mathrm{NJ}$ ) at locations $0^{\circ}, 22.5^{\circ}, 45^{\circ}, 65.5^{\circ}$, and $90^{\circ}$ from the stimulation point. Compared with the peak temperature at the site of heating $\left(82^{\circ} \mathrm{C}\right)$, at $22.5^{\circ}$ radially from the stimulation point the temperature declined to $45^{\circ} \mathrm{C}$, and by $45^{\circ}$ (the radial interval between the eight stimulation sites), the temperature had declined to well below nociceptor threshold $\left(32^{\circ} \mathrm{C}\right)$, verifying that the stimulus primarily excited local thermoreceptors. In more recent studies using a similar protocol (Bauer et al., 2000), lack of sensitization or habituation (Carstens and Wilson, 1993) was verified over six consecutive trials (versus only three in this study), also spaced 32 $\min (4 \mathrm{~min} / \mathrm{stimulus} \times$ eight stimulus sites $)$ apart.

Tail withdrawal responses were characterized by direction and speed of response. A ring $(3.5 \mathrm{~cm}$ in diameter for intact animals and $2.5 \mathrm{~cm}$ for spinalized rats) was positioned around the tail $30 \%$ of tail length caudal to the base of the tail $(\sim 6 \mathrm{~cm})$. The ring was inscribed with a polar coordinate system in $10^{\circ}$ increments in which the ventral aspect of the rat was assigned $0^{\circ}$ and progressed clockwise around the tail (see Fig. 1) (the coordinate system is shown below the tail). The direction of response was measured by visually noting the point on the inside of the ring where the tail made contact. To measure movement speed, a thin, moist sponge was placed on the inside of the ring, and a thin silver wire with a small blob of electrode paste was positioned to just touch the tail on the side opposite to the expected direction of movement. These connections were incorporated into an electrical, voltage-divider circuit that was configured to detect the time of onset of movement and time of contact with the ring, thus allowing calculation of average movement speed. Movement times ranged from 10 to $90 \mathrm{msec}$, corresponding to $5-50 \mathrm{~cm} / \mathrm{sec}$. For each rat, response speeds were normalized by the average speed across all 24 trials. In most experiments $(n=17)$, both direction and speed were measured, but in some additional experiments $(n=10)$, only direction was measured.

The direction and speed, measured as described above, were used to calculate vectored responses for each stimulus, with the polar angle corresponding to direction and polar magnitude corresponding to speed. The results were analyzed and displayed as either individual (see Fig. $4 C, D$ ) or average (see Figs. 2, 3). Averages were then calculated in two ways. Average vectors (see Fig. 2) were calculated by averaging the individual Cartesian $x$ and $y$ coordinates ("true" vector average). Average magnitudes and directions (see Fig. 3) were calculated by averaging the individual directions and speeds. Thus, responses that have large magnitude but variable direction (ventral stimulus, $0^{\circ}$, in spinalized rats) will have small magnitude vector averages (see Fig. $2 A, 0^{\circ}$ ) but large average magnitudes (see Fig. 3, spinal, $0^{\circ}$ ).

In some experiments, the rat was spinalized $24 \mathrm{hr}$ before the experiment. The rat was anesthetized with sodium pentobarbital (Nembutal, 50 $\mathrm{mg} / \mathrm{kg}$; Abbott, North Chicago, IL), and the spinal cord at vertebral T6-T7 (spinal segment T7) was exposed by laminectomy and incision of the dura. After coagulating superficial blood vessels and applying local anesthetic (4\% lidocaine, 5-10 $\mu$; Roxane, Columbus, $\mathrm{OH}$ ) both superficially and deep, the spinal cord was cut with Castroviejo scissors. Completeness of section was verified visually. The incision was closed, 5 $\mathrm{ml}$ of saline was administered (subcutaneously), and the rat was monitored until recovered. The site of spinalization, spinal T6, was rostral to the location of motoneurons that participate in the tail withdrawal response (Brink and Pfaff, 1980; Grossman et al., 1982; Cargill, 1983).

Each of the eight locations was stimulated three times. The stimulation sequence was dorsal, right-dorsal, right, right-ventral, ventral, left-ventral, left, and left-dorsal $\left(0^{\circ}, 45^{\circ} \ldots 315^{\circ}\right)$, with the three successive stimuli delivered at each adjacent location 4 min apart (or $32 \mathrm{~min}$ apart for stimuli delivered to the same site) to avoid habituation or sensitization (Carstens and Wilson, 1993). Rats were killed by an overdose of sodium pentobarbital $(200 \mathrm{mg} / \mathrm{kg})$. Data were analyzed and plotted using MATLAB, Excel, Sigmaplot, SPSS, and Coreldraw. Statistical comparisons between movement directions and speeds (see Fig. 3) were made using one-way ANOVA with a Tukey post hoc test. Comparisons to $0^{\circ}$ in Figure $4, A$ and $B$, were made with one-way $t$ tests and a Holms sequential Bonferroni correction for

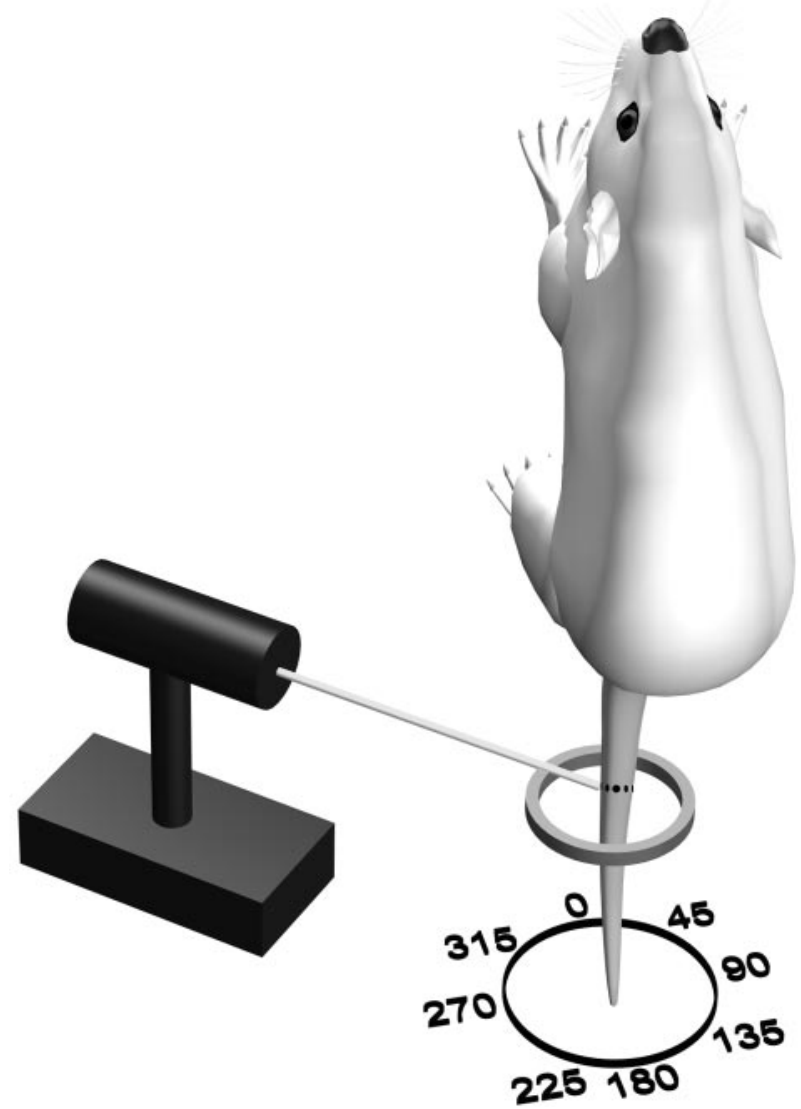

Figure 1. Experimental arrangement. The diagram illustrates the experimental methods, including the stimulus, direction, and speed measurement ring, and coordinate system for measurements. Ventral corresponds to $0^{\circ}$, which increases clockwise around the tail. To apply stimuli to different locations on the tail, the rat, measurement ring, and coordinate system were rotated. Black circles on the tail indicate stimulus targets.

multiple comparisons (Green et al., 1997). $\alpha$ was set to 0.05. All error bars are SEM.

Experiments conformed to the National Institutes of Health and International Association for the Study of Pain guidelines for animal care and were approved by the Institutional Animal Care and Use Committee at James Madison University.

\section{RESULTS}

Pinpoint heat stimuli were applied to eight circumferentially located points around the tail (Fig. 1). Figure 2 shows the average response vector for each rat (thin arrows) and the mean response vector (thick arrows; length doubled for clarity) for all rats at each of the eight radial locations. Response vectors directed toward the center of the circle correspond to responses directly away from the stimulus, whereas responses directed perpendicularly outward would correspond to responses into the stimulus. In this representation, Sherrington's proposed constant, ventral flexion response (one form of the categorical pattern) would correspond to identical response vectors all pointing upwards. In contrast, the continuous hypothesis would predict that the vectors would all point toward the center of the circle.

\section{Spinalized rats exhibited a ventral bias}

The results obtained from spinalized rats and shown in Figure $2 \mathrm{~A}$ revealed a hybrid pattern of responses. Overall, the response vectors were directed primarily inward, consistent with the continuous hypotheses. In only a few instances were the response 


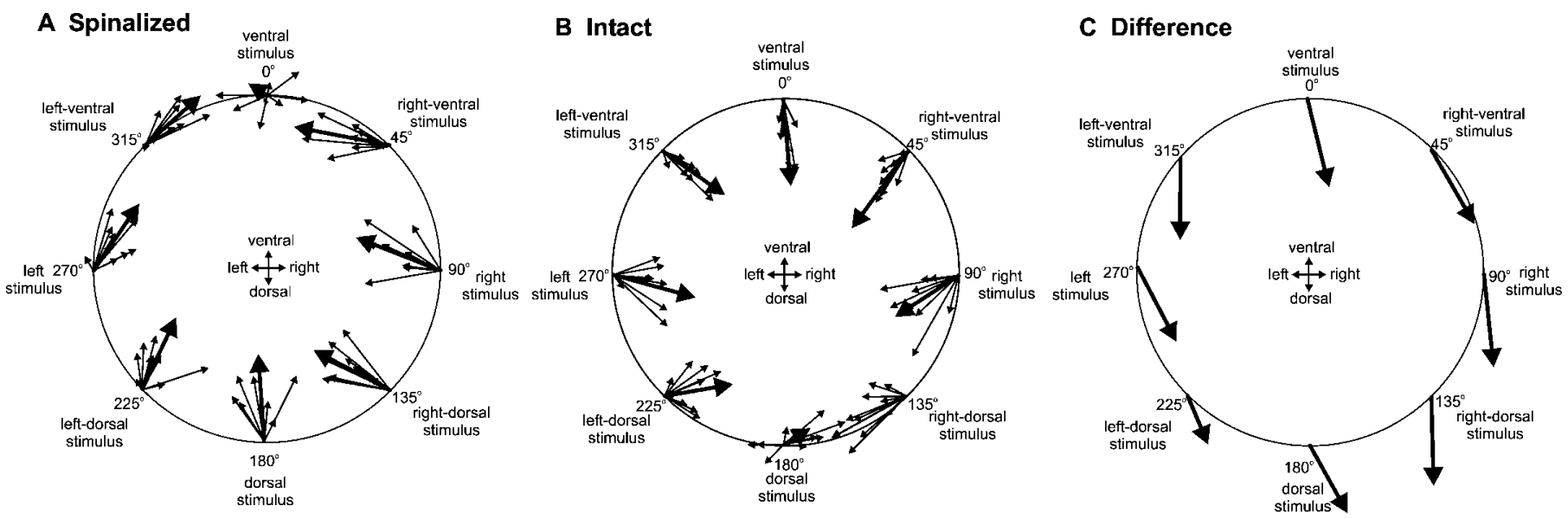

Figure 2. Response vectors. Each panel shows average responses from each rat (thin arrows; each is a vector average of three trials) and the mean response for all eight (spinalized) or nine (intact) rats (thick arrows). The length of the mean vector is doubled for clarity. The circle represents the circumference of the tail, with the origin of each set of arrows positioned at the point at which the heat stimulus was delivered. Thus, arrows pointing directly toward the center correspond to movements directly away from the stimulus, whereas arrows pointing $180^{\circ}$ away from the center (outward) correspond to movement into the stimulus. $A$, Spinalized rats. Responses are primarily toward the center but with a pronounced ventral bias. $B$, Intact rats. Responses are also primarily toward the center, but the bias is now in the dorsal direction. $C$, Difference. The vector difference between the intact and spinal mean responses reveals that they differ by an approximately constant magnitude dorsal movement vector.

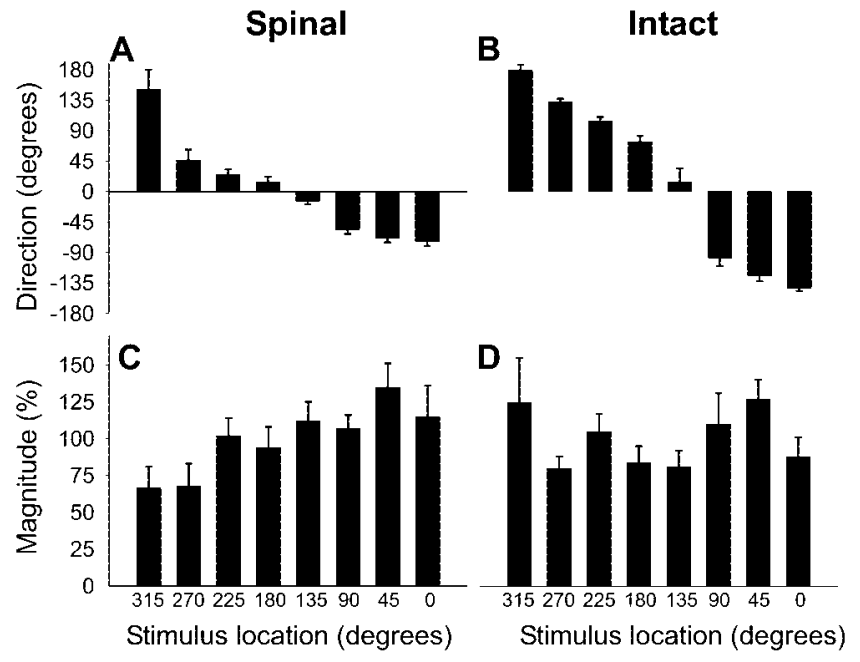

Figure 3. Direction and magnitudes of responses. Each bar is the average \pm SEM of the directions and magnitudes of individual responses. Thus, they differ somewhat from the average responses in Figure 1 because the mean responses in Figure 1 are the mean vectors rather than the means of individual directions and magnitudes shown in this Figure (the difference is only large for $0^{\circ}$ in the spinalized and $180^{\circ}$ in the intact rats). $A$, Direction-spinalized. Response directions differ significantly $(p<0.05)$ for 19 of 28 pairwise comparisons. B, Direction-intact. Response directions differ significantly $(p<0.05)$ for 20 of 28 pairwise comparisons. $C$, Magnitude-spinalized. Each trial was normalized by the average response in that rat previous to averaging across rats. Response directions differ significantly $(p<0.05)$ for only 2 of 28 pairwise comparisons. D, Magnitudes-intact. Response directions do not differ significantly $(p<0.05)$ for any pairwise comparisons.

vectors directed outward toward the stimulus. Figure $3 A$, which quantifies the directions of responses, verifies that the response directions depended significantly on stimulus location. Magnitude (speed of response), however, did not significantly vary with stimulus location (Fig. 3C).

In addition to the overall inward direction, the responses exhibited a pronounced bias, or "tilt", in the ventral direction. The ventral bias is most readily seen as an upward tilt for stimuli applied to the left $\left(270^{\circ}\right)$ and right $\left(90^{\circ}\right)$ sides. Figure $4 A$ better depicts the ventral bias by showing the difference between the observed direction of response and a hypothetical response directly inward; deviation from $0^{\circ}$ thus indicates the magnitude of the ventral (upward) or dorsal (downward) bias. Individually, five of the six lateral stimuli (dorsal and ventral stimuli are omitted) exhibited a significant ventral bias. Although the ventral bias was significant for most lateral stimulus locations, the magnitude of the bias increased as the stimulus location became more ventral. The responses to pure dorsal and ventral stimuli also reflected a marked ventral bias; dorsal stimuli produced a consistent, ventrally directed response, whereas ventral stimuli evoked a highly variable response (see below).

The pattern of responses to ventral stimuli showed a striking qualitative difference in pattern in comparison to the other seven stimulus locations. The directions of response to ventral stimuli were highly variable, including some average responses directly toward the stimulus; other stimulus locations resulted in a tight cluster of directions (Fig. $2 A$ ), although the average magnitudes were similar (Fig. $2 C$ ). The nature of the underlying process is better seen in Figure $4 C$, which shows the directions of response for all 42 individual trials (typically three per rat; in comparison, each of the eight vectors in Figure $2 A$ is a vectored average of the three individual trials). Again, dorsal stimulation resulted in a moderately tight cluster of responses, whereas the responses to ventral stimulation were widely scattered. Especially noteworthy are the pronounced clusters of responses leftward, ventrally (into the stimulus) and, to a lesser degree, rightward. Highly different responses were sometimes seen between different trials in the same rat, although responses within individual rats were usually similar.

\section{Intact rats exhibited a dorsal bias}

Withdrawal reflexes in spinalized rats must arise from spinal reflexes. In contrast, responses in intact rats could arise from spinal reflexes identical to those in spinalized rats, supraspinal, "long-loop" responses, and/or spinal reflexes under tonic descending modulation. To assess the role of supraspinal control of 


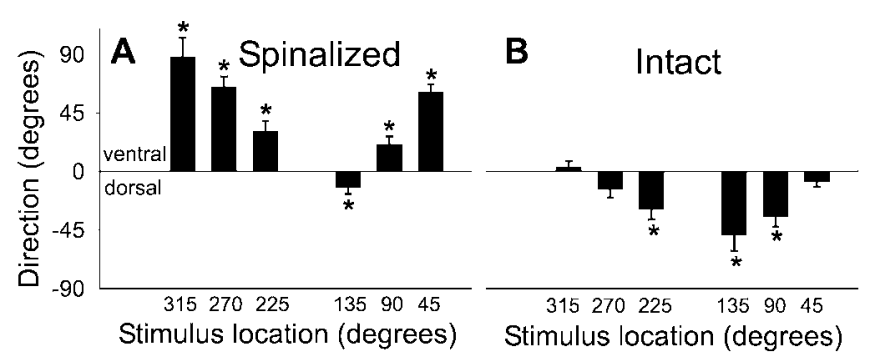

C Spinalized

D Intact
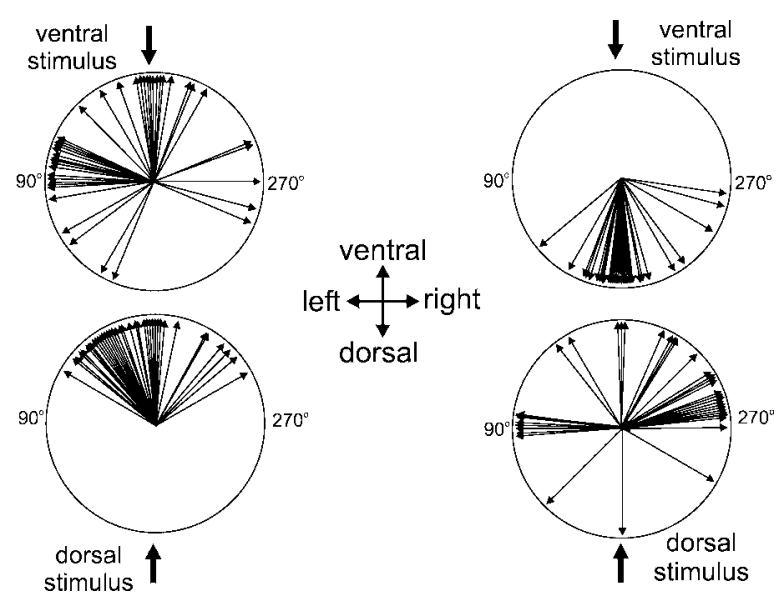

Figure 4. In $A$ and $B$, average difference in direction between the actual response and a hypothetical response directed toward the center (directly away from the stimulus). Thus, if the continuous hypothesis was correct, all bars would be at $0^{\circ}$. Purely ventral $\left(0^{\circ}\right)$ and dorsal $\left(180^{\circ}\right)$ stimuli are omitted. $A$, Spinalized. Ventral ( $n=5$, upward) or dorsal (downward, $n=$ $\left.1,135^{\circ}\right)$ bias are significantly different from $0^{\circ}(p<0.005)$ for all. $B$, Intact. Dorsal (downward) bias is significant $(p<0.005)$ for 4 of 6 stimulus. In $C$ and $D$, all response vectors (13 or 14 rats, $\sim 3$ per rat) are graphed with unity magnitude to illustrate the distribution of directions. Identical vector are shifted by $1^{\circ}$ for clarity. Responses to two of the eight stimulus locations are included; ventral on top and dorsal on the bottom. $C$, Spinalized. Dorsal stimulus evokes a tight cluster of responses in the ventral direction, whereas ventral stimulus evokes highly variable responses with clusters in the left, ventral, and to lesser degree, right direction. $D$, Intact. Ventral stimulus evokes a cluster of responses in the dorsal direction, whereas dorsal stimulus evokes highly variable responses with clusters in the left and right directions.

the tail withdrawal reflex, similar experiments were performed with intact, unanesthetized rats.

Figure $2 B$ shows that there were similarities and differences between the spinalized and intact rats. Like spinalized rats, responses were primarily directed inward, away from the stimulus. Figure $3 B$ shows that the dependence on location was statistically significant, whereas Figure $3 D$ again shows that the magnitude of individual responses did not depend on stimulus location. In further similarity, intact rats also exhibited a bias in the direction of response that also increased as the stimulus location approached the direction of the bias. In sharp contrast, however, the bias in intact rats was exactly opposite to that in spinalized rats; that is, the bias was in the dorsal direction. Figure $4 B$ shows that the bias was individually significant for three of six lateral stimulus locations. The pattern of individual responses shown in Figure $4 D$ for dorsal and ventral stimuli were also inverted in comparison to the responses in the spinalized rat. Furthermore, the clustering of lateral responses in the left $\left(270^{\circ}\right)$ or right $\left(90^{\circ}\right)$ directions is especially clear, but in contrast to the spinalized rats, the cluster of responses directed into the stimulus (dorsal direction in this case) is absent.

\section{Supraspinal pathways contributed a constant dorsal response}

The net influence of supraspinal pathways is shown in Figure $2 C$, which is the vector difference between the mean responses in spinalized (Fig. 2A) and intact (Fig. 2B) rats. All eight vectors are highly similar in both direction and magnitude around the dorsal direction. Thus, the inclusion of supraspinal pathways "adds" a constant, dorsally directed response to the spinal response at all stimulus locations.

\section{DISCUSSION}

The results from this study demonstrate that in the spinalized rat, pinpoint heat stimuli applied to the tail evoke withdrawal responses that have two spatial components; one directly away from the stimulus and the other in the ventral direction. Furthermore, stimuli applied to the ventral surface, which "should" evoke a response in the dorsal direction, instead evoke responses that are highly variable but primarily in the lateral directions. In contrast, in intact rats the component directed away from the stimulus persists, but the ventral bias is replaced by a dorsal bias, whereas the response to ventral stimuli becomes more variable and lateral. Thus, the addition of supraspinal pathways appears to invert the ventrodorsal component without altering the left-right component of the response.

Previous studies in several nonmammalian species have provided evidence for both the continuous (Stein et al., 1986; Eaton and Emberley, 1991; Lewis and Kristan, 1999) and categorical (Krasne and Wine, 1984; Stein et al., 1986) pattern. For the mammalian leg withdrawal reflex, early studies by Sherrington (1910) and Lundberg (1979) on the flexor reflex afferent concept supported the categorical pattern by showing that the responses to stimuli in different locations were primarily similar flexion responses. In contrast, Hagbarth (1952) and more recently Schouenborg et al. (1994) have presented data consistent with a continuous pattern in both spinalized (Schouenborg et al., 1992) and intact, halothane-anesthetized (Schouenborg and Kalliomaiki, 1990) rats. Their results showed electromyographic activity (EMG) patterns consistent with the stimulated portion of the limb being moved away from the stimulus. In the tail there is limited and conflicting evidence (Carstens and Wilson, 1993; Holmberg and Schouenborg, 1996; Levinsson et al., 1999). Our results are not consistent with either pattern, and instead suggest the spatial transformation for the tail withdrawal reflex is a hybrid continuous-categorical strategy in both intact and spinalized rats.

There are several possible explanations for the difference between our results and those obtained in the thorough series of investigations by Schouenborg's group. First, the neural circuitry controlling the tail may differ from the leg. Second, they studied anesthetized intact (Schouenborg and Kalliomaiki, 1990) and acute spinalized (5-12 hr) (Schouenborg et al., 1992) rats, whereas we used intact awake rats and short-term chronic (24 hr) rats. Third, they characterized the withdrawal responses in terms of EMG and inferred the direction of movement based on the pattern and magnitude of muscle EMG, although there is brief mention of a three-dimensional motion analysis system (Schouenborg et al., 1994) used to express movement relative to the surface geometry of the limb. Because of the biomechanical complexity of the leg musculature, accurate prediction of direction and magni- 
tude could be difficult. Further studies will be needed to evaluate the source of the differences.

Supraspinal descending pathways that influence spinal reflexes arise from both motor and sensory regions of the brain. The descending motor pathways include the corticospinal, vestibulospinal, reticulospinal, and rubrospinal pathways (Schwindt, 1981). The descending somatosensory pathways include corticospinal and several bulbospinal pathways (Gebhart, 1988). Descending pathways been shown to influence limb and tail responses by either modulating spinal reflexes (Schwindt, 1981) or superimposing an additional "long-loop" response mediated by the cortex, cerebellum, or other supraspinal structures (Lee et al., 1983). Our results showed that spinalization caused a marked alteration in direction, with the bias switching from ventral in spinalized rats (flexion) to dorsal (extension) in intact rats. In terms of a Cartesian coordinate system, this amounts to an alteration of the ventrodorsal component without effect on the left-right component, raising the possibility that the two perpendicular components of movement may be under independent neural control. Similarly, a change in direction in response to a distant nociceptive stimulus was also reported by Morgan and Whitney (1996) and Morgan (1999), which may have been mediated by descending pathways. Although our results shed little light on whether the direction of the reflex bias is altered by modulation of existing spinal reflexes or addition of a supraspinal component, the similarity of the results in intact and spinalized rats (except for reversal of the bias) suggest that the basic features may be encoded in the spinal cord. Similarly, we can only speculate on which descending motor or sensory pathways may be involved. Because spinalization could be interpreted as removing an extensor bias, the vestibulospinal pathway, which has been shown to facilitate extension and inhibit flexion (Schwindt, 1981), is especially indicated.

A striking finding in both intact and spinalized rats was the pattern of response to stimuli applied opposite to the preferred direction; that is, dorsal stimuli in intact and ventral stimuli in spinalized rats. Responses were highly variable, occasionally occurring into the stimulus, and clustered in the left and right directions rather than the expected ventral or dorsal directions. One of many possible explanations is that the neural strategy for stimuli in these locations is strictly categorical, requiring the nervous system choose one of a limited number of responses. Alternatively, the mapping may be continuous but highly nonlinear, such that a small shift in stimulus position results in dramatically different directions of response. Combined with some lack of reproducibility in stimulus positioning between trials and animals, this could primarily explain the pattern of response.

In both intact and spinalized rats, stimuli applied to each of the eight circumferential locations evoked responses with unique directions (Fig. 3A,B), suggesting a unique pattern of sensory input associated with each location. However, previous studies of the mechanical sensitivity of sacral spinal interneurons that may mediate the tail withdrawal reflex showed that the interneurons had bilateral mechanical receptive fields on the tail (Laird and Cervero, 1989; Douglass and Carstens, 1997), and thus would not be able to distinguish between left and right stimuli. However, it is unknown whether the effects of heat stimuli on sacral interneurons are also bilateral (Mitchell and Hellon, 1977; Douglass and Carstens, 1987). Furthermore, there is no direct evidence that the interneurons studied were necessary and sufficient to induce the tail withdrawal reflex. Other unstudied interneurons could have provided the unilateral thermal sensitivity needed for the unique mapping of response direction to stimulus location.

Our results raise the evolutionary question of how the observed spatial pattern of response may benefit the animal. In general, the continuous strategy in an ideal world would be best for removing the body part from the stimulus. However, a categorical strategy may be advantageous because of biomechanical or environmental asymmetries, and also reduces the degrees of freedom (Giszter et al., 1993), thereby simplifying and possibly speeding the movement. The rat tail is normally kept on or close to the ground, making strict ventral movements impossible; our results in the intact rat thus showed the expected dorsal bias. The clustering of lateral responses to ventral stimulation also makes sense because the extent of movement in the dorsal direction is limited, and a full lateral movement would bring the tail as close as possible to the body, providing greater protection from injury. Although speculative, these ideas suggest that supraspinal control of the rat tail may have evolved to better adapt the response to the biomechanical and environment constraints on tail movement.

\section{REFERENCES}

Bauer RE, Belyea BC, Gentile SM, Welsford IG, Cleland CL (2000) Spatial transformations in the withdrawal reflex of the rat tail. Soc Neurosci Abstr 26:57.17.

Bernstein N (1967) The coordination and regulation of movements. New York: Pergamon.

Bizzi E, Mussa-Ivaldi FA, Giszter S (1991) Computations underlying the execution of movement: a biological perspective. Science 253:287-291.

Brink EE, Pfaff DW (1980) Vertebral muscles of the back and tail of the albino rat (Rattus norvegicus albinus). Brain Behav Evol 17:1-47.

Cargill CL (1983) The tail flick reflex in the rat. Master's thesis, University of Texas at Galveston.

Carstens E, Wilson C (1993) Rat tail flick reflex: magnitude measurement of stimulus-response function, suppression and habituation. J Neurophysiol 70:630-639.

Dubner R (1994) Methods of assessing pain in animals. In: Textbook of pain (Wall PD, Melzack R, eds), pp 293-314. Edinburgh: Churchill Livingstone.

Douglass DK, Carstens E (1997) Responses of rat sacral spinal neurons to mechanical and noxious thermal stimulation of the tail. J Neurophysiol 77:611-620.

Eaton RC, Emberley DS (1991) How stimulus direction determines the trajectory of the mauthner-initiated escape response in a teleost fish. $\mathrm{J}$ Exp Biol 161:469-487.

Gebhart GF (1988) Descending inhibition of nociceptive transmission. In: Basic mechanisms of headache (Olesen J, Edvinsson L, eds), pp 201-212. New York: Elsevier.

Giszter SF, Mussa-Ivaldi FA, Bizzi E (1993) Convergent force fields organized in the frog's spinal cord. J Neurosci 13:467-491.

Green SB, Salkind NJ, Akey TM (1997) Using SPSS for Windows. New Jersey: Prentice-Hall.

Grossman ML, Basbaum AI, Fields HL (1982) Afferent and efferent connections of the rat tail flick reflex (a model used to analyze pain control mechanisms). J Comp Neurol 206:9-16.

Hagbarth K-E (1952) Excitatory and inhibitory skin areas for flexor and extensor motoneurones. Acta Physol Scand 94:1-58.

Holmberg H, Schouenborg J (1996) Postnatal development of nociceptive withdrawal reflexes in the rat: a behavioral and electromyographic study. J Physiol (Lond) 493:239-252.

Krasne FB, Wine JJ (1984) The production of tailflip escape responses. In: Neural mechanisms of startle behavior (Eaton RC, ed), pp 179-212. New York: Plenum.

Lee RG, Murphy JT, Tatton WG (1983) Long-latency myotatic reflexes in man: mechanisms, functional significance, and changes in patients with Parkinson's disease or hemiplegia. Adv Neurol 39:489-508.

Levinsson A, Luo XL, Holmberg H, Schouenborg J (1999) Developmental tuning in a spinal nociceptive system: effects of neonatal spinalization. J Neurosci 19:10397-10403.

Lewis JE, Kristan WB (1998) Quntitative analysis of a directed behavior in the medicinal leech: implications for organizing motor output. J Neurosci 18:1571-1582.

Lundberg A (1979) Multisensory control of spinal reflex pathways. Prog Brain Res 50:11-28:11-28.

Mitchell D, Hellon RF (1977) Neuronal and behavioural responses in rats during noxious stimulation of the tail. Proc R Soc Lond B Biol Sci 197:169-194. 
Morgan MM (1999) Paradoxical inhibition of nociceptive neurons in the dorsal horn of the rat spinal cord during a nociceptive hindlimb reflex. Neuroscience 88:489-498.

Morgan MM, Whitney PK (1996) Behavioral analysis of diffuse noxious inhibitory controls (DNIC): antinociception and escape reactions. Pain 66:307-312.

Norekian TP, Satterlie RA (1996) Whole body withdrawal circuit and its involvement in the behavioral hierarchy of the mollusk clione limacina. J Neurophysiol 75:529-537.

Saltzman E (1979) Levels of sensorimotor representation. J Math Psychol 20:91-163.

Schouenborg J, Kalliomaiki J (1990) Functional organization of the nociceptive withdrawal reflexes. I. Activation of hindlimb muscles in the rat. Exp Brain Res 83:67-78.

Schouenborg J, Holmberg H, Weng H (1992) Functional organization of the nociceptive withdrawal reflexes. II. Changes of excitability and receptive fields after spinalization in the rat. Exp Brain Res 90:469-478.

Schouenborg J, Weng H, Holmberg H (1994) Modular organization of spinal nociceptive reflexes: a new hypothesis. News Physiol Sci 9:261-265.

Schwindt PC (1981) Control of motoneuron output by pathways descending from the brain stem. In: Handbook of behavioral neurobiology, Vol 5, Motor coordination (Towe EL, Luschei ES, eds), pp 139-230. New York: Plenum.

Sherrington CS (1910) Flexion-reflex of the limb, crossed extensionreflex, and reflex stepping and standing. J Physiol (Lond) 40:28-121.

Stein PSG, Camp AW, Robertson GA, Mortin LI (1986) Blends of rostral and caudal scratch reflex motor patterns elicited by simultaneous stimulation of two sites in the spinal turtle. J Neurosci 6:2259-2266. 